\title{
Heldenreisen wie bei Humboldt?
} Das Storytelling in der Wissenschaft

\author{
Christoph Koch
}

Am 27. Dezember 1831 sticht der Forscher in See. Er geht auf eine jahrelange Fahrt, deren theoriegeschichtliche Folgen unabsehbar sind und sich später als weltstürzend erweisen sollten. Der Enthusiasmus des Aufbruchs ist indes getrübt von hartnäckiger Seekrankheit. Es ist ein überwältigendes vegetatives Unbehagen, das den 22-Jährigen auf die Einsicht in seine (nach damals weithin unumstößlicher Auffassung) geschöpfliche Natur zurückwirft. Heute dürfen wir sogar sagen: zurückwirft auf das Tier in Charles Darwin, dessen empfindlicher Magen an das Rollen und Krängen des Dreimasters „HMS Beagle“ nicht adaptiert ist. Offen steht dem an das Schiff gefesselten Forschungsreisenden allerdings die Flucht vor seinem Elend in erzählte Welten: Darwin zieht sich auf ein Sofa zurück und studiert die Reiseberichte Alexander von Humboldts, die in gleich zwei Ausgaben an Bord sind.

Der Preuße, als reisender Forscher, furchtloser Entdecker und akribisch Dokumentierender, inspiriert den britischen Studienabbrecher. Humboldt erreicht dies nicht so sehr als Dozent gelehrter Vorträge, als Publizist wissenschaftlicher Original-Aufsätze oder als Mitglied einer gelehrten Akademie (der Königlich Preußischen Akademie der Wissenschaften, seit 1805), sondern als Erste-Person-Erzähler eines „Personal Narrative“ (PN) - so der

\footnotetext{
C. Koch $(\triangle)$

Gruner + Jahr GmbH, Hamburg, Deutschland

E-Mail: koch.christoph@stern.de
} 
geläufige Titel der englischen Übersetzung ${ }^{1}$. Bereits auf der ersten Seite des Vorwortes zum ersten der sieben Bände des PN kann Darwin aus der Feder der Übersetzerin Humboldts, Helen Maria Williams, lesen: „The narratives of travellers, and, above all, the description of those remote countries of the globe, which have immortalized the name of Cook, have always had a particular attraction for my mind [...]" (Humboldt und Bonpland 1814, S. v).

"The narratives of travellers": Darwin wie Humboldt wollen von der Natur und ihrer aufsuchenden Enträtselung erzählt bekommen. Entsprechend erzählen sie dann auch selbst, Humboldt enthusiastisch, Darwin zurückgenommener - und sie stellen sich damit in eine Traditionslinie, die bis heute ungebrochen ist.

Antje Boëtius beispielsweise, Tiefseebiologin, Leiterin des Alfred-Wegener-Instituts in Bremerhaven und Trägerin des Communicator-Preises der Deutschen Forschungsgemeinschaft, sagte kürzlich dem Deutschlandfunk: „Wenn ich im U-Boot abtauche, dann gehöre ich zu den wenigen Menschen, die jemals die Tiefsee persönlich erkundet haben - es sind ja weniger als Menschen, die im All waren - und dann ist das Publikum schon mal gefesselt, da steht jetzt eine Frau, war im U-Boot, ist in die größten Tiefen abgetaucht, und wenn es da ein Loch im U-Boot gegeben hätte, wäre sie jetzt tot, und trotzdem macht die das." (Krieger 2019) Übrigens, dies nur nebenbei, fehlt auch in dieser Forscherinnenbiografie das Motiv der Brechreiz erregenden Seekrankheit auf erster großer Fahrt nicht.

Humboldt jedenfalls, jüngst durch eine erzählerisch meisterliche Biografie von Andrea Wulf (2016) repopularisiert, hätte Boëtius womöglich gern zugehört; seine zeitgenössische englische Übersetzerin jedenfalls hat Humboldts hybrider Erzähl- und Erkenntnisprosa (s. u.) programmatische Sätze vorangestellt, die quasi inhaltsgleich vorwegnehmen, was uns heutige Storytelling-Fans nachdrücklich empfehlen:

Happy the traveller with whom the study of nature has not been merely the cold research of the understanding, in the explanation of her properties, or the solution of her problems! Who, while has interpreted her laws, has adored her sublimity, and followed her steps with passionate enthusiasm, amidst that

\footnotetext{
${ }^{1}$ Vollständig: „Personal Narrative of Travels to the Equinoctial Regions of the New Continent During the Years 1799-1804" (Humboldt und Bonpland 1814). Das französische Original ist unter dem Titel „Relation Historique du voyage aux régions équinoxiales du Nouveau Continent" ab 1814 in Paris erschienen. Der Naturforscher Aimé Bonpland (1773-1858) war Humboldts Reisegefährte und Co-Autor.
} 
solemn and stupendous scenery, those melancholy and sacred solitudes, where she speaks in a voice so well understood by the mysterious sympathy of the feeling heart. (Humboldt und Bonpland 1814, S. vi f.)

Tatsächlich handelt es sich bei den Reiseberichten prominenter Naturforscher des „zweiten Entdeckungszeitalters“ - neben Humboldt unter den Deutschsprachigen etwa bei Georg Forster und Adelbert von Chamisso um „hybride Texte“, „welche die drei Gelehrten nicht nur an die Experten und Patrone der Naturwissenschaften um 1800, sondern auch und in erster Linie an das allgemeine Lesepublikum ihrer Zeit adressierten", wie Johannes Görbert (2014, S. 4) herausgearbeitet hat: In die Chronologie der Erzählung betten sie empirische Beobachtungen aus Geologie, Biologie oder Ethnologie ein.

Dieser Modus der Textgestaltung wird von den Verfassern nicht zwingend exklusiv gewählt; am anderen Ende des Spektrums ihres Schreibens publizieren auch vorviktorianische Forscher bereits explizite und formal strenge Fachbeiträge für eine emergente scientific community von Insidern. Jedoch gilt: „Überall dort, wo die Berichte über die Forschungsexpeditionen die Struktur von Rhizomen annehmen, die mehrere, sonst häufig nach Disziplinen getrennte Diskurssegmente umfassen, ist viel eher eine ,literarischere Gestaltung anzunehmen als dort, wo die Reisetexte sich auf einen Beitrag zu enger umgrenzten Fachbereichen kaprizieren." (Görbert 2014, S. 14). Die Frage ist nun: Hat sich das im Zuge der „Entzauberung der Welt“ (Max Weber), im Rahmen ihrer „Verwandlung“ (Jürgen Osterhammel) grundlegend verändert? Darwins Publikation seiner bereits auf der Beagle antizipierten Evolutionslehre stellt immerhin einen wesentlichen Fixpunkt der Vertreibung des Menschen aus dem Mittelpunkt der Welt dar. ${ }^{2}$

Derzeit erscheint es so, als sei der Bedarf an Erzähltem aus der Wissenschaft einst wie damals groß. Nachdem sich die Wissenschaft mehr als ein Jahrhundert lang professionalisiert, fachlich und sprachlich differenziert und damit auch um die Behauptung ihres Autonomieraums gerungen hat, wächst gegenwärtig offensichtlich die Nachfrage nach derlei hybriden Texten wieder - nach

\footnotetext{
${ }^{2}$ Patrick Breitenbach, Wissenschaftsblogger und Innovationstreiber bei ZDF Digital, argumentierte im Interview mit Wissenschaft im Dialog auf der Folie exakt dieser Entwicklung 2017 entschieden „pro Storytelling": „Was früher magisch und mythisch war, macht die Wissenschaft kaputt, indem sie aufklärt. Das ist in vielerlei Hinsicht unbestritten gut und wichtig, aber durch diese Form der Entzauberung verlieren die Menschen am Ende auch wieder etwas. Es entstehen dabei Lücken, die gefüllt werden müssen, weil Menschen fantasiebegabte Wesen sind, die sich ihre Wirklichkeit selbst erschaffen (Konstruktivismus). Und das Zauberhafte ist für uns offenbar sehr wichtig." (Wissenschaft im Dialog 2017).
} 
narrativen Konstruktionen nämlich, die die universelle Zugänglichkeit zum Feld der Wissenschaft durch Leseführung und durch Faszination erhöhen, dabei jedoch, so der Kern des Anspruchs, gültige Evidenz und empirisch Gesichertes darbieten. Ihre LeserInnen wollen diese Texte binden und führen. 1985 - 121 Jahre nachdem Darwin mit der Copley Medal ihre höchste Auszeichnung empfangen hat - ist es die Royal Society, die in ihrem Report „The Public Understanding of Science" einen bis heute fortwirkenden Impuls dazu gibt. Walter Bodmer und Kollegen postulieren in dieser einflussreichen Expertise:

Scientists must learn to communicate with the public, be willing to do so, and indeed consider it their duty to do so all scientists need, therefore, to learn about the media and the constraints and learn how to explain science simply, without jargon and without being condescending. Each sector of the scientific community should consider, for example, providing training and communication and greater understanding of the media, arranging non-specialist lectures and demonstrations, organizing scientific competitions for younger people, providing briefings for journalists and generally by improving their public relations. (The Royal Society 1985, S. 6)

Seither ist die Debatte um das „Wie“ und „Wieviel“ der Wissenschaftskommunikation nicht mehr abgerissen. Zu Marksteinen der Entwicklung zählen in Deutschland etwa das PUSH-Memorandum von 1999 (Hochschulrektorenkonferenz 1999), die Gründung von Wissenschaft im Dialog (WiD) und des Informationsdiensts Wissenschaft (idw) auf Senderseite, aber auch in letzter Zeit die des deutschen Science Media Center (SMC) als „einfordernder" Struktur und Dienstleister für einen unabhängigen Wissenschaftsjournalismus.

Es fehlt hier an Raum, die einschlägigen Expertisen und Kontroversen der jüngsten Vergangenheit zu rekapitulieren; ermutigend ist allerdings, wie lebendig sich das Gespräch über die Kommunikation der Wissenschaft mit der Öffentlichkeit entwickelt hat. Die wissenschaftlichen Akademien haben mit ihren Gutachten dazu beigetragen, der Siggener Kreis als unabhängiges Forum der Wissenschaftskommunikatoren mit Positionsbestimmungen, Empfehlungen und Leitlinien-Arbeit ${ }^{3}$, ebenso der Wissenschaftsjournalismus, der seiner teils bedrohten wirtschaftlichen Situation zum Trotz ebenfalls aktiv an der gegenwärtigen Debatte teilhat.

${ }^{3}$ Publikationen herunterladbar unter: https://www.wissenschaft-im-dialog.de/projekte/siggener-kreis/. 
Auch am Bekenntnis zur Stärkung der Wissenschaftskommunikation und an Appellen zum Kompetenzerwerb innerhalb des Wissenschaftssystems fehlt es auch von höchster politischer Ebene nicht. Bundesforschungsministerin Anja Karliczek etwa erklärte im September 2018: „Wissenschaftlerinnen und Wissenschaftler, die bereits stark in der Kommunikation sind, sollten dafür mehr Anerkennung erfahren. Auch können verbesserte Aus- und Weiterbildungsangebote zur Stärkung der Kommunikationskompetenz beitragen. Mir liegt die Wissenschaftskommunikation am Herzen, denn ich bin überzeugt, dass sie dazu beiträgt, das Vertrauen in und das Interesse an der Wissenschaft in ihrer ganzen Breite und damit auch in unsere Demokratie weiter zu stärken." (BMBF 2018). Hier tritt es schließlich in offizieller Verlautbarung ans Licht, das Hoffnungsmotiv, das einschlägige Kommunikationserfordernisse politisch begründet: Das Vertrauen in die Institutionen, letztlich gar in die demokratische Ordnung, die sie rahmt, werde gestärkt, wenn WissenschaftlerInnen von sich, von ihrer Arbeit und ihren Resultaten erzählen.

Und ohne Zweifel: Das Ringen um Aufmerksamkeit hat sich mit der großen Vielfalt von sozialen und klassischen Medien im World Wide Web stark intensiviert, und die öffentliche Intervention zahlreicher WissenschaftlerInnen in vielen Ländern bei den „Marches for Science“ gegen Missbrauch und Bedrohung der wissenschaftlichen Methode und ihres Debattenethos markiert Dringlichkeit. Die Frage bleibt: Wie sollen WissenschaftlerInnen sprechen? Im Ton eines - mit den Worten der Humboldt-Übersetzerin Williams - auktorialen „cold research of the understanding“ der Welt (was sie vor dem Verdacht der Parteinahme schützen könnte)? Oder unter Heraufbeschwörung der „mysterious sympathy of the feeling heart“, wie es sich für TiefseeforscherInnen nach einer Tauchfahrt anbietet?

Der Trend der letzten Jahre ist hier also eindeutig: Forschende jeglicher Provenienz sollen tatsächlich von sich erzählen; sie sollen sich des "Storytellings“ bedienen, um (so die Annahme) in breiten Öffentlichkeiten Vertrauenskapital zu bilden. Dies, das sei im nahen zeitlichen Umfeld des Presse-Skandals um den Spiegel-Reporter Claas Relotius pflichtschuldigst angemerkt, immer unter der Voraussetzung, dass das als real Erzählte aus dem Forscherleben nicht fiktional, nicht fiktionalisiert ist. ${ }^{4}$ Die Mittel des Geschichtenbaus jedoch, so

\footnotetext{
${ }^{4}$ Auch diese Gefahr, wie offenbar auch das Prinzip des kognitiven Konstruktivismus, war Menschen des 19. Jahrhunderts bewusst. Wilhelm Busch schrieb 1886 in seiner Autobiografie: „Immer, wenn man was wissen will, muss man sich auf die zweifelhafte Dienerschaft des Kopfes und der Köpfe verlassen und erfährt nie recht, was passiert ist. Wer ist heutigen Tages noch so harmlos, dass er Weltgeschichten und Biographien für richtig hält? Sie gleichen den Sagen und Anekdoten, die Namen, Zeit und Ort benennen, um sich glaubhaft zu machen. Sind sie unterhaltlich erzählt, sind sie ermunternd und lehrreich, oder rührend und erbaulich, nun gut! So wollen wir's gelten lassen." (Busch 1988, S. 9).
} 
propagieren seine Anhänger, die sie auch lehrend an WissenschaftskommunikatorInnen und WissenschaftlerInnen vermitteln, seien nicht nur legitim, sondern Voraussetzung guter Kommunikationswirkung.

Dass dieses Argument Gewicht hat, lässt sich sogar an Textsorten belegen, die dem eigenen Anspruch nach offensiv verhehlen, dass es sich bei ihnen um Erzähltes handeln könnte: Am Korpus der wissenschaftlichen Literatur selbst. So haben Hillier et al. (2016) gezeigt, dass zumindest für das gegenwärtig hoch kompetitive Feld der Klimawissenschaften gilt: Je stärker sich im Abstract einer Studie narrative Elemente nachweisen lassen, umso häufiger wird sie zitiert. Wer zu erzählen weiß, kann somit offenbar nicht nur profitieren, wenn einmal wieder Wissenschaft-in-die-Öffentlichkeit gefordert ist, sondern auch direkt, innerhalb der professionellen Community:

Our findings are consistent with the prevailing understanding across a range of fields that audiences tend to understand and recall narratives - that is, stories - far better than information received in other ways. The result is surprising, though, in the context of professional scientific communication, in which expository styles dominate the published literature, word counts are strictly limited by editorial policies, graphics are routinely used to present results, and citation frequency is often considered to depend largely - even primarily upon the strength of the science. (Hillier et al. 2016)

Provokant ließe sich anmerken: Es deutet sich an, dass die Erkenntnisse der Rezeptionsforschung auch von innerhalb der Forschung unhintergehbar sind, so stark sie sich auch in einem formalen Purismus "typischer" Wissenschaftssprache ergehen mag. Der beobachtete Effekt steigert sich übrigens noch mit dem Impact Factor des einschlägigen Journals.

Mit einiger Wahrscheinlichkeit ist es unter Forschenden und in ihren Institutionen längst tacit knowledge, ist implizit bekannt, dass erzählende Textgestaltung, und sei sie noch so zurückgenommen, wirkt. Mehr noch: Sofern sie innerhalb der Wissenschaftsgemeinde wirkt, dort also, wo die toposrelevanten Abstraktionsfähigkeit am stärksten ausgebildet ist, wie viel stärker wird der Überzeugungseffekt zugunsten eigener Anliegen und Botschaften dann womöglich erst in der breiten Öffentlichkeit sein?

Die Annahme, in narrativen Formen das ideale Werkzeug gewinnender Kommunikation gefunden zu haben, hat jedenfalls die Management- und PR-Literatur in den vergangenen Jahren geradezu überschwemmt. $\mathrm{Zu}$ den einschlägigen Top-Titeln bei Amazon zählen Handbücher für Geschichtenerzähler in PR und Marketing, aber auch für Personaler, die mit Storys Konflikte lösen und Motivation stiften sollen. Natürlich gibt es Storytelling-Fibeln 
für Multimedia, Podcast und für Dummies. Als Crashkurse, „mit Daten“, für „Leadership“ und CEOs, für Journalisten (Lampert und Wespe 2017; bereits in vierter Auflage) - und sehr gern auch gestützt auf „Hirnforschung“. All dies klingt so unmittelbar plausibel, so simpel und doch vielversprechend, dass auch ich mich der Versuchung nicht entziehen mochte, Ihnen von Darwin und von Humboldt zu erzählen, eine Brücke zu spannen in eine Zeit, von der wir annehmen, dass die strenge Ausformung der wissenschaftlichen Textsorten noch nicht gegeben war, in der es noch reichlich Unentdecktes gab und viel zu tun. Also mehr Geschichtenstoff?

Systemtheoretiker würden wohl sagen: Die funktionale Differenzierung des Wissenschaftssystems war in den 1830er-Jahren noch nicht so weit vollzogen, dass es seine Grenzen und Eigenlogiken auch im Feld seiner sprachlichen Konventionen noch nicht hinreichend abgezirkelt hatte. Heute dagegen seien Peer Review, die Wissenschaftssprache Englisch, die fachspezifischen Jargons und der formale Aufbau von Papers so weit etabliert, dass Geschichtenerzählen allenfalls in der Beziehung zur Umwelt, letztlich also in der Gewinnung der Herzen von Stakeholdern und Laien, seinen Platz fände. Dass das empirisch falsch ist, haben wir eben gesehen; dass es jedoch auch innerhalb der Fachwissenschaft erhebliche Zweifel an der universalen Zweckmäßigkeit und Sinnhaftigkeit forscherischer Heldenreisen und ergreifender Institutionen-Storys gibt, müssen wir jetzt begründen.

Zur Eröffnung des 11. Forums Wissenschaftskommunikation, am 7. November 2018, sprach Julika Griem, Vizepräsidentin der Deutschen Forschungsgemeinschaft, Leiterin des Kulturwissenschaftlichen Instituts Essen, Anglistin und Erzählforscherin, die Keynote. Die Arena der medialisierten Gegenwartsgesellschaft charakterisierte sie wie folgt: „Hier gerieren sich einerseits Zahnpastawerbung, der Wetterbericht und auch schon mal Fußballtrainer als forschungsnah, während andererseits immer neue Formate der Unterhaltung durch Wissenschaft kreiert werden." (Griem 2018, S. 2). Griems Intervention (ich kann es bezeugen, denn ich war in Bonn zugegen und führte das anschließende Podiumsgespräch mit Julika Griem, darf also an dieser Stelle von neuem erzählend in Erscheinung treten) übte auf einen Gutteil der Versammelten einen Überraschungseffekt aus, einige nachfolgende Reaktionen waren entschieden ablehnend.

Griem führte unter anderem aus:

- Wissenschaftskommunikation sei „in einigen ihrer dominanten Tendenzen zu einseitig ausgerichtet" (S. 2).

- Wesentlicher Grund dafür sei die Dramatisierung praktisch jeglichen erdenklichen Stoffes, oft im Stile der „berühmten und vielfach 
nachgeahmten Naturdokumentationen der BBC“; entsprechend „folgen wir mutigen Forscherinnen und Forschern auf ihren von einem raunenden Erzählduktus und schwellenden Orchesterklängen unterlegten Reisen an die ,last frontiers' der Biodiversität und ihre tragischen Konfliktlagen“" (S. 2).

- Die Folge sei eine „Narrativisierung“ und „Eventisierung insbesondere von Forschung" (S. 3).

- Folglich finde eine Verdrängung der mühseligen, Ausdauer fordernden Wirklichkeit von Forschung durch Heroisierung statt. Helden, meist männlich, erobern die Welt; die Mühen der Ebene, der Anträge, der Planstellenbewirtschaftung, der Dokumentation, der Gremien, bleiben unsichtbar: „Akten, Daten und Apparate sind deutlich schwerer zu heroisieren als Individuen." (S. 5)

- Strukturen und Prozesse, auch „die Sorgfalt, die gerade auch für unspektakuläre Phänomene aufgebracht werden muss, um wissenschaftliches Wissen methodisch nachvollziehbar legitimieren zu können“, fänden somit keine angemessene Berücksichtigung in der Kommunikation. (S. 5)

Aus wissenschaftsjournalistischer Sicht - und damit aus einer Perspektive, die erzählerische ebenso wie nachrichtenförmige Formate in einer längeren Tradition parallel nutzt, als es die institutionelle Wissenschaftskommunikation tut - erscheint eine pauschale Zurückweisung der Kritik Julika Griems am Storytelling-Trend nicht angemessen. ${ }^{5}$ Tatsächlich, so kann man zwar einwenden, dürfte es schwierig sein, in der breiten

\footnotetext{
${ }^{5}$ Auf der Diskussionsveranstaltung „Relotius Reloaded“, die am 1. Februar 2019 im Kulturwissenschaftlichen Institut Essen stattfand, hat Griem in diesem Kontext präzisiert: „Ich wollte hier nur einwerfen, dass, wenn ich über Storytelling spreche, ich eine ganz bestimmte Weise des Erzählens meine. Nämlich eine extrem simplifizierte, spezifische Versprechung durch sehr einfache, eingängige Erzählschemata. In der Branche redet man über die Heldenreise, den fünf Punkten, die dieser Held immer auf gleiche Weise zurücklegt; Sie kennen diese Abenteuererzählungen, die dann dabei rauskommen: Aufbruch, Bewährung, Heimkehr. Dieses Muster wird an Filmschulen für Drehbuchautoren, für Journalisten, gelehrt und auf diese Weise verbreitet. Es wird zum Beispiel Schülern vermittelt, wenn sie Bewerbungstraining schon in der Schule bekommen: ,Inszeniere Dich als ein Held eines eigenen Lebens, mach' Storytelling, denn das macht dein Leben für Arbeitgeber viel attraktiver'. Diese Art des Erzählens ist eine ganz spezifische; und es gibt viele andere Arten des Erzählens, die komplizierter, interessanter sind und die bestimmte Widersprüche, Paradoxien der Wirklichkeit auf ganz andere Weise verarbeiten. Und mein Anliegen wäre es, zu untersuchen, wie dieser Kult des Storytelling, diese Fetischisierung einer bestimmten Form von Erzählen in so viele Bereiche eingesickert ist, nämlich auch in die Wissenschaftskommunikation. Auch wir bekommen gesagt: ,Jetzt machen Sie doch mal eine schöne Story aus ihrer spröden Philologie.“" (eigene Mitschrift).
} 
Öffentlichkeit Aufmerksamkeitsschwellen zu überwinden, indem man einen DFG-Antrag zum Subjekt eines Schicksalsberichtes macht und seine einzelnen Begutachtungsschritte akribisch dokumentiert.

Weiterhin haben wir es in der multimedialisierten Aufmerksamkeitsökonomie mit Wettbewerbern zu tun, die - wie die Framing-Forschung belegt - die Narrativisierung und Dramatisierung permanent als Mittel ihrer Überzeugungsarbeit nutzen. So stützt sich etwa die Bewegung der „Impfgegner“, jüngst von der Weltgesundheitsorganisation zur globalen Gesundheitsgefahr erklärt, konsequent auf anekdotische Evidenz, also auf Storys von vermeintlichen Impfschäden. Sie bettet diese in Großerzählungen verschwörungsförmiger Kartelle aus Politik und pharmazeutischer Industrie ein und gewinnt Anhänger über intensive Nutzung sozialer Medien (Chirico 2017). Donzelli et al. (2018) haben jüngst eine große Zahl einschlägiger YouTube-Videos analysiert. Sie zeigen auf, wie intensiv sich antiszientistische Kommunikation auf Storytelling stützt; sie greift dabei nicht nur die institutionalisierte Wissenschaft an, sondern - wesentlich fundamentaler - den wissenschaftlichen Denkstil selbst, das wissenschaftliche Ethos sowie etablierte Gütekriterien der Forschung. Entsprechend den Befürchtungen, denen mit den „Marches of Science“ begegnet werden sollte, könnte es hier geboten sein, dem „schwarzen“ Storytelling antiwissenschaftlicher Agitatoren mit „weißem“ Storytelling entgegenzutreten - einfach deshalb, weil es eine Intervention ist, für deren Wirksamkeit es gute Evidenz gibt (s. o.). Für ihren Spezialfall schlagen Donzelli et al. (2018) daher vor: Öffentliche Institutionen (also auch Universitäten und Wissenschaftsorganisationen) „should be more engaged in establishing a web presence in order to provide reliable information, answers, stories, and videos so to respond to questions of the public about vaccination." (Abstract, Hervorhebung von mir).

Unmittelbar vom Forum Wissenschaftskommunikation hat Jan Steffen im Blog von Wissenschaft im Dialog zudem gefragt: „Wollen wir nur Menschen erreichen, die ohnehin offen sind für geistige Herausforderungen?“ (Steffen 8. November 2018). Diese Frage ist selbstredend rhetorisch; Steffen führt aus: „Wissenschaftskommunikation[,] wie sie ihr [Griem] vorschwebt, setzt ein interessiertes Publikum voraus, das bereit ist, sich, sanft überfordern zu lassen'“" Er fährt fort:

Dabei dürfen wir aber nicht vergessen, dass wir Menschen nicht zwingen können, sich mit Wissenschaft zu beschäftigen. Aus meiner Ausbildung als Tageszeitungsjournalist weiß ich, wie schnell Menschen das Interesse an einem Text verlieren. Schon ein Wort, das Leserinnen oder Leser nicht verstehen, kann dazu führen, dass sie „aussteigen“ und den Text nicht weiterlesen. Auf die 
Bandbreite der Wissenschaftskommunikation übertragen bedeutet das: Schon kleinste Barrieren können Menschen abschrecken. Sie wenden ihre Aufmerksamkeit statt der Wissenschaft dann (im besten Fall) lieber dem belanglosen Krimi oder (im schlimmsten Fall) simplen populistischen Thesen zu. (Steffen 8. November 2018)

Mit dieser noch jungen Kontroverse ist ein Anstoß zu einer Debatte gegeben, die eine intensive Fortführung wert ist. Die letzten Jahre haben gezeigt, dass sich die Wissenschaftskommunikation zunehmend reflexiv organisiert; sie diskutiert über die Professionen - Pressestellen, PR-Organisationen, Wissenschaftsjournalismus, Akademien - hinweg über Sinn und Werte, aber eben auch über Formen und Formate ihrer letztlich den Grad gegenseitigen und öffentlichen Vertrauens widerspiegelnden Aktivitäten.

Anzumerken ist, in Ergänzung der Hoffnung, dass dieser Diskurs intensiv fortgeführt werde, noch: Es gibt gute Belege dafür, dass Storytelling tatsächlich nicht immer und überall die Methode der Wahl sein wird. Der spontan ersichtliche Impact einer wissenschaftlichen Entdeckung spielt eine wesentliche Rolle dafür, inwieweit legitime Dramatisierung das Mittel der Wahl im Ringen um Aufmerksamkeit ist. Auch dies belegt ein Beispiel aus der Geschichte der modernen Biologie.

Es ist 1953, James Watson und Francis Crick haben am Cavendish-Laboratory in Cambridge die DNA-Doppelhelix entdeckt - ein akut nobelpreisverdächtiger Durchbruch, ist doch der Mechanismus der Selbstvervielfältigung genetischer Information bislang unverstanden und die Brücke zwischen Evolutionstheorie und Genetik nicht vollständig geschlagen. Das revolutionäre Potenzial ihrer Molekülcharakterisierung verhehlen die Forscher jedoch mit den Worten: „It has not escaped our notice that the specific pairing that we have postulated immediately suggests a possible copying mechanism for the genetic material." (Watson und Crick 1953, S. 737). Entscheidend für unseren Kontext ist: Diese beinahe karikaturhafte Überzeichnung lakonischer Wissenschaftssprache im Gewand britischen Understatements war für Eingeweihte dennoch unmittelbar als sensationell zu decodieren - und in vielen Fällen vermutlich auch „realphysiologisch“ atemberaubend: „Biologists everywhere recognized the finding as world-changing." (Martinez-Conda und Macknik 2017, S. 8129). Sie hätten, darauf war Verlass, passende Storys bereits im Kopf. Und sie würden sie jedem erzählen - das war ebenso wenig zu vermeiden wie im Gefolge der Publikation der Gedanken, zu denen Charles Darwin auf der Beagle gefunden hatte. 


\section{Literatur}

BMBF. (2018). Pressemitteilung: 090/2018. Bundesforschungsministerin Anja Karliczek sagt anlässlich des Wissenschaftsbarometers. https://www.bmbf.de/de/ bundesforschungsministerin-anja-karliczek-sagt-anlaesslich-des-wissenschaftsbarometers-7018.html. Zugegriffen: 26. Jan. 2019.

Busch, W. (1988). Von mir über mich. In W. Busch (Hrsg.), Sämtliche Werke II. München: Bertelsmann (Erstveröffentlichung 1886).

Chirico, F. (2017). Vaccinations and media: An on-going challenge for policy makers. Journal of Health and Social Sciences, 2(1), 9-18.

Donzelli, G., Palomba, G., Federigi, I., Aquino, F., Cioni, L., Verani, M., et al. (2018). Misinformation on vaccination: A quantitative analysis of YouTube videos. Human Vaccines \& Immunotherapeutics, 14(7), 1654-1659.

Görbert, J. (2014). Die Vertextung der Welt: Forschungsreisen als Literatur bei Georg Forster, Alexander von Humboldt und Adelbert von Chamisso. Berlin: De Gruyter.

Griem, J. (2018). Zumutungen. Wissenschaftskommunikation und ihre Zumutungen. (Keynote auf dem Forum Wissenschaftskommunikation 2018). http://www.dfg. de/download/pdf/dfg_im_profil/reden_stellungnahmen/2018/181107_keynote_ fwk18_griem.pdf. Zugegriffen: 20. Jan. 2019.

Hillier, A., Kelly, R. P., \& Klinger, T. (2016). Narrative style influences citation frequency in climate change science. PLoS ONE, 11(12), e0167983.

Hochschulrektorenkonferenz. (1999). Memorandum „Dialog Wissenschaft und Gesellschaft".https://www.hrk.de/positionen/beschluss/detail/memorandum-dialogwissenschaft-und-gesellschaft/. Zugegriffen: 23. Jan. 2019.

Krieger, A. (2019). Der Forscher als Held und Abenteurer. https://www.deutschlandfunkkultur.de/storytelling-in-der-wissenschaft-der-forscher-als-heldund.976.de.html?dram:article_id=437369. Zugegriffen: 20. Jan. 2019.

Lampert, M., \& Wespe, R. (2017). Storytelling für Journalisten. Wie baue ich eine gute Geschichte? Köln: Herbert von Halem.

Martinez-Conde, S., \& Macknik, S. L. (2017). Opinion: Finding the plot in science storytelling in hopes of enhancing science communication. Proceedings of the National Academy of Sciences, 114(31), 8127-8129.

Steffen, J. (8. November 2018). Wollen wir nur Menschen erreichen, die ohnehin offen sind für geistige Herausforderungen? https://www.wissenschaft-im-dialog. de/blog/blogartikel/beitrag/wollen-wir-nur-menschen-erreichen-die-ohnehin-offen-sind-fuer-geistige-herausforderungen/. Zugegriffen: 23. Jan. 2018.

The Royal Society. (1985). The public understanding of science. Report of a Royal Society ad hoc Group endorsed by the Council of the Royal Society. https://royalsociety.org/ /media/royal_society_content/policy/publications/1985/10700.pdf. Zugegriffen: 21. Jan. 2019. 
von Humboldt, A., \& Bonpland, A. (1814). Personal narrative of travels to the equinoctial regions of the new continent, during the years 1799-1804. Übersetzt ins Englische von H. M. Williams. London: Longman et al. Digitalisat bei Google Books.

Watson, J. D., \& Crick, F. H. (1953). Molecular structure of nucleic acids. Nature, 171, 737-738.

Wissenschaft im Dialog. (2017). „Wissenschaftskommunikatoren müssen Zauber in die Vermittlung bringen" - Patrick Breitenbach über Storytelling in der Wissenschaft. https://www.wissenschaft-im-dialog.de/blog/blogartikel/beitrag/ wissenschaftskommunikatoren-muessen-zauber-in-die-vermittlung-bringenpatrick-breitenbach-ueber-s/. Zugegriffen: 17. Jan. 2019.

Wulf, A. (2016). Alexander von Humboldt und die Erfindung der Natur. München: Bertelsmann.

Christoph Koch, Diplom-Humanbiologe, B.Sc. (Hons.) (Social Sciences), MBA (Life Sciences). Geboren 1967, beim stern als Leitender Redakteur für das Wissenschaftsressort zuständig. Seit 2018 zudem Redaktionsleiter von Dr. v. Hirschhausens stern Gesund leben. Seit Abschluss der Henri-Nannen-Schule 1997 Wissenschaftsjournalist, Mitglied in diversen Gremien, darunter im WissensWerte-Programmbeirat, dem Kuratorium der Weisse Liste gGmbH und dem Kuratorium der Stiftung Warentest. Gemeinsam mit Mai Thi Nguyen-Kim und Lisa Ruhfus Moderator von „Die Debatte“ bei Wissenschaft im Dialog.

Open Access Dieses Kapitel wird unter der Creative Commons Namensnennung 4.0 International Lizenz (http://creativecommons.org/licenses/by/4.0/deed.de) veröffentlicht, welche die Nutzung, Vervielfältigung, Bearbeitung, Verbreitung und Wiedergabe in jeglichem Medium und Format erlaubt, sofern Sie den/die ursprünglichen Autor(en) und die Quelle ordnungsgemäß nennen, einen Link zur Creative Commons Lizenz beifügen und angeben, ob Änderungen vorgenommen wurden.

Die in diesem Kapitel enthaltenen Bilder und sonstiges Drittmaterial unterliegen ebenfalls der genannten Creative Commons Lizenz, sofern sich aus der Abbildungslegende nichts anderes ergibt. Sofern das betreffende Material nicht unter der genannten Creative Commons Lizenz steht und die betreffende Handlung nicht nach gesetzlichen Vorschriften erlaubt ist, ist für die oben aufgeführten Weiterverwendungen des Materials die Einwilligung des jeweiligen Rechteinhabers einzuholen.

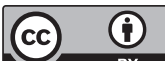

